\title{
Social Setting
}

National Cancer Institute

\section{Source}

National Cancer Institute. Social Setting. NCI Thesaurus. Code C102712.

The surroundings or environment in which social activities occur. 